IJMMS 25:11 (2001) 727-743

PII. S0161171201005154

http://ijmms.hindawi.com

(c) Hindawi Publishing Corp.

\title{
$H$-FUNCTION WITH COMPLEX PARAMETERS II: EVALUATION
}

\section{FADHEL A. AL-MUSALLAM and VU KIM TUAN}

(Received 10 November 1998 and in revised form 17 December 1999)

ABSTRACT. Sufficient conditions for computation of the $H$-functions with complex parameters by means of residues are derived and some examples are given.

2000 Mathematics Subject Classification. Primary 33C60.

1. Introduction. Let

$$
k(s)=\frac{\prod_{j=1}^{P} \Gamma\left(p_{j}+\alpha_{j} s\right)}{\prod_{j=1}^{Q} \Gamma\left(q_{j}+\beta_{j} s\right)} .
$$

Here $P$ and $Q$ are nonnegative integers, $p_{j}(1 \leq j \leq P), q_{j}(1 \leq j \leq Q)$ are complex numbers, and $\alpha_{j}(1 \leq j \leq P), \beta_{j}(1 \leq j \leq Q)$ are nonzero complex numbers. Throughout the present paper an empty product is interpreted as unity and an empty sum as zero.

Let $c$ be a real number such that $c \neq \operatorname{Re}\left(-p_{j} / \alpha_{j}\right)$, for all $j$ with $\operatorname{Re} \alpha_{j}=0$, and $\Lambda_{c}$ denote a contour from $c-i \infty$ to $c+i \infty$ not passing through any pole of $k(s)$ and such that

(1) if $\operatorname{Re} \alpha_{j}>0$, then all the points

$$
s=-\frac{m}{\alpha_{j}}-\frac{p_{j}}{\alpha_{j}}, \quad m=0,1,2, \ldots,
$$

are to the left of $\Lambda_{c}$.

(2) If $\operatorname{Re} \alpha_{j}<0$, then all the points (1.2) are to the right of $\Lambda_{c}$.

(3) If $\operatorname{Re} \alpha_{j}=0$, and $\operatorname{Re}\left(-p_{j} / \alpha_{j}\right)<c$, then all the points (1.2) are to the left of $\Lambda_{c}$.

(4) If $\operatorname{Re} \alpha_{j}=0$, and $\operatorname{Re}\left(-p_{j} / \alpha_{j}\right)>c$, then all the points (1.2) are to the right of $\Lambda_{c}$.

The authors in [1] introduced the notion of $H$-function with complex parameters as a Mellin-Barnes type integral [2] as follows,

$$
H_{Q}^{P}\left(z, c \mid \begin{array}{l}
(p, \alpha)_{P} \\
(q, \beta)_{Q}
\end{array}\right)=\frac{1}{2 \pi i} \int_{\Lambda_{c}} k(s) z^{-s} d s .
$$

Here, and elsewhere in this paper, $(p, \alpha)_{P}=\left(p_{1}, \alpha_{1}\right),\left(p_{2}, \alpha_{2}\right), \ldots,\left(p_{P}, \alpha_{P}\right)$, and similarly for $(q, \beta)_{Q}$.

When $(\alpha)_{P}$ and $(\beta)_{Q}$ are integer vectors, the $H$-function with complex parameters reduces to the Meijer $G$-function (see $[4,11,12,13,14,15,16]$ ), and when $(\alpha)_{P}$ and $(\beta)_{Q}$ are real vectors, it reduces to the Fox $H$-function $[5,19]$. The $G$ - and $H$-functions play an important role in statistics and physical sciences (see [7, 8, 9, 10, 19]). 
In [1], the authors derived necessary and sufficient conditions, under which the integral (1.3) defining the $H$-function converges absolutely. The approach in [1] was based on the following asymptotic expansion of $|k(s)|$.

LEMMA 1.1. For $\operatorname{Re} s \geq 0$, and as $|s| \rightarrow \infty$,

$$
\begin{aligned}
|k(s)| \simeq & Y(s)|s|^{\Delta_{0}} e^{T(\ln |s|) \operatorname{Re} s} e^{-\mu(\ln |s|) \operatorname{Im} s} e^{(\operatorname{Im} s)(\mu-M-T \operatorname{Arg} s)} \\
& \times e^{(\operatorname{Re} s)\left[X-L-T-\mu \operatorname{Arg} s+(\pi / 2) \operatorname{sign}(\operatorname{Im} s)\left(\sum_{\operatorname{Re} \alpha_{j}=0} \operatorname{Im} \alpha_{j}-\sum_{\operatorname{Re} \beta_{j}=0} \operatorname{Im} \beta_{j}\right)\right]} \\
& \times \frac{\prod_{j=1}^{Q}\left|(1 / 2)\left(1+S\left(\beta_{j}, s\right)\right)+\left(1-S\left(\beta_{j}, s\right)\right) \sin \pi\left(q_{j}+\beta_{j} s\right)\right|}{\prod_{j=1}^{P}\left|(1 / 2)\left(1+S\left(\alpha_{j}, s\right)\right)+\left(1-S\left(\alpha_{j}, s\right)\right) \sin \pi\left(p_{j}+\alpha_{j} s\right)\right|},
\end{aligned}
$$

where

$$
\begin{aligned}
& \Delta_{0}=-\frac{1}{2}(P-Q)+\sum_{j=1}^{P} \operatorname{Re} p_{j}-\sum_{j=1}^{Q} \operatorname{Re} q_{j} \\
& \mu=\sum_{j=1}^{P} \operatorname{Im} \alpha_{j}-\sum_{j=1}^{Q} \operatorname{Im} \beta_{j}, \quad T=\sum_{j=1}^{P} \operatorname{Re} \alpha_{j}-\sum_{j=1}^{Q} \operatorname{Re} \beta_{j}, \\
& M=\sum_{j=1}^{P}\left[\operatorname{Arg}\left(\alpha_{j} \operatorname{sign}\left(\operatorname{Re} \alpha_{j}\right)\right) \operatorname{Re} \alpha_{j}+\left(\ln \left|\alpha_{j}\right|\right) \operatorname{Im} \alpha_{j}\right] \\
& -\sum_{j=1}^{Q}\left[\operatorname{Arg}\left(\beta_{j} \operatorname{sign}\left(\operatorname{Re} \beta_{j}\right)\right) \operatorname{Re} \beta_{j}+\left(\ln \left|\beta_{j}\right|\right) \operatorname{Im} \beta_{j}\right], \\
& L=\sum_{j=1}^{P}\left[\left(\operatorname{Arg} \alpha_{j}\right) \operatorname{Im} \alpha_{j}-\left(\ln \left|\alpha_{j}\right|\right) \operatorname{Re} \alpha_{j}\right]-\sum_{j=1}^{Q}\left[\left(\operatorname{Arg} \beta_{j}\right) \operatorname{Im} \beta_{j}-\left(\ln \left|\beta_{j}\right|\right) \operatorname{Re} \beta_{j}\right], \\
& X=\pi\left(\sum_{\operatorname{Re} \alpha_{j}<0}\left|\operatorname{Im} \alpha_{j}\right|-\sum_{\operatorname{Re} \beta_{j}<0}\left|\operatorname{Im} \beta_{j}\right|\right)+\frac{\pi}{2}\left(\sum_{\operatorname{Re} \alpha_{j}=0}\left|\operatorname{Im} \alpha_{j}\right|-\sum_{\operatorname{Re} \beta_{j}=0}\left|\operatorname{Im} \beta_{j}\right|\right), \\
& S(\alpha, s)= \begin{cases}-(\operatorname{sign} \operatorname{Im} \alpha) \operatorname{sign} \operatorname{Im} s, & \operatorname{Re} \alpha=0, \\
\operatorname{sign} \operatorname{Re} \alpha, & \operatorname{Re} \alpha \neq 0,\end{cases} \\
& Y(s)=(2 \pi)^{(P-Q) / 2} \frac{\prod_{j=1}^{P}\left|\alpha_{j}\right|^{-(1 / 2)+\operatorname{Re} p_{j}}}{\prod_{j=1}^{Q}\left|\beta_{j}\right|^{-(1 / 2)+\operatorname{Re} q_{j}}} e^{-m(s)},
\end{aligned}
$$

where

$$
\begin{aligned}
m(s)= & -\frac{\pi}{2}(\operatorname{sign} \operatorname{Im} s)\left(\sum_{\operatorname{Re} \alpha_{j}=0} \operatorname{Im} p_{j}-\sum_{\operatorname{Re} \beta_{j}=0} \operatorname{Im} q_{j}\right)+\left(\sum_{j=1}^{P} \operatorname{Im} p_{j}-\sum_{j=1}^{Q} \operatorname{Im} q_{j}\right) \operatorname{Arg} s \\
& +\sum_{\operatorname{Re} \alpha_{j} \neq 0} \operatorname{Arg}\left(\alpha_{j} \operatorname{sign} \operatorname{Re} \alpha_{j}\right) \operatorname{Im} p_{j}-\sum_{\operatorname{Re} \beta_{j} \neq 0} \operatorname{Arg}\left(\beta_{j} \operatorname{sign} \operatorname{Re} \beta_{j}\right) \operatorname{Im} q_{j} .
\end{aligned}
$$

Here we write $f(z) \simeq g(z)$, as $|z| \rightarrow \infty, \alpha \leq \arg z \leq \beta$, if $f(z)=g(z)[1+o(1)]$, as $|z| \rightarrow \infty$, within the sector $\alpha \leq \arg z \leq \beta$. 
The following theorem gives the conditions, derived in [1], under which the integral (1.3) defining the $H$-function converges absolutely.

THEOREM 1.2. If

$$
N=\sum_{j=1}^{P}\left|\operatorname{Re} \alpha_{j}\right|-\sum_{j=1}^{Q}\left|\operatorname{Re} \beta_{j}\right|,
$$

and $M, T$, and $\mu$ are as in Lemma 1.1, then the integral (1.3) converges absolutely if and only if,

(1) $\mu=0, N>0$, and for $|\arg z-M|<(\pi / 2) N$, or

(2) $\mu=0, N \geq 0, \arg z=M \pm(\pi / 2) N$, and $\Delta_{c}=\Delta_{0}+c T<-1$.

These conditions, naturally, reduce to those of the classical case where the $\alpha_{j}$ 's and $\beta_{j}$ 's are real. Also in [1] some well-known integrals that are not special cases of the classical Fox $H$-function were expressed as $H$-function with complex parameters.

The present paper is a continuation of the work in [1]. In this paper, we determine sufficient conditions that enable one to compute the $H$-function with complex parameters (1.3) as a sum of residues at the right or the left poles of $k(s) z^{-s}$. It should be mentioned that Braaksma [3] was the first to apply the residue theorem to evaluate the integral (1.3) in the special case when $\alpha_{j}= \pm 1$ and $\beta_{j}= \pm 1$. See also [17, 18], where the residue theorem was used to compute the integral (1.3), with real $\alpha_{j}$ 's and $\beta_{j}$ 's, for two other contours. It is striking that under some conditions, the $H$-function with complex parameters can be computed, in certain annulus of the complex plane, by the residues at the right poles as well as the residue at the left poles. This phenomenon has no parallel when the vectors $\left(\alpha_{j}\right)_{P}$ and $\left(\beta_{j}\right)_{Q}$ are real vectors. We apply our result to compute the $H$-function (1.3) in some particular cases.

2. Evaluation by means of residues. We need the following remark.

REMARK 2.1. If $w$ is a complex number, that is, not a zero of $\sin z$, and $z_{0}$ is the zero of $\sin z$ closest to $w$, then

$$
|\sin w| \geq \min \left\{\frac{1}{\pi}\left|w-z_{0}\right|, \frac{1}{4}\right\} e^{|\operatorname{Im} w|} .
$$

To see this, observe that $|\sin x| \geq(2 / \pi)|x|$, for all $-\pi / 2 \leq x \leq \pi / 2$. Since $z_{0}$ is the zero of $\sin z$ closest to $w$, then necessarily, $\left|\operatorname{Re}\left(w-z_{0}\right)\right| \leq \pi / 2$, and moreover,

$$
\begin{aligned}
|\sin w|^{2} & =\left|\sin \left(w-z_{0}\right)\right|^{2}=\sin ^{2} \operatorname{Re}\left(w-z_{0}\right)+\sinh ^{2} \operatorname{Im}\left(w-z_{0}\right) \\
& \geq\left(\frac{2}{\pi}\right)^{2} \operatorname{Re}^{2}\left(w-z_{0}\right)+\operatorname{Im}^{2}\left(w-z_{0}\right) \geq \frac{4}{\pi^{2}}\left|w-z_{0}\right|^{2} .
\end{aligned}
$$

If $|\operatorname{Im} w|>\ln 2$,

$$
|\sin w| \geq|\sinh \operatorname{Im} w| \geq \frac{1}{2}\left(e^{|\operatorname{Im} w|}-1\right)=\frac{1}{2} e^{|\operatorname{Im} w|}\left(1-e^{-|\operatorname{Im} w|}\right)>\frac{1}{4} e^{|\operatorname{Im} w|} .
$$

If $|\operatorname{Im} w| \leq \ln 2$, then (2.2) implies

$$
\frac{1}{\pi}\left|w-z_{0}\right| e^{|\operatorname{Im} w|} \leq \frac{2}{\pi}\left|w-z_{0}\right| \leq|\sin w| .
$$

Combining (2.3) and (2.4) yields (2.1). 
Let $\Omega_{c}$ denote a contour from $c-i \infty$ to $c+i \infty$ not passing through any pole of the function $k(s)$, defined by (1.1). Denote by $\mathrm{PO},(\mathrm{PO})_{r}$, and $(\mathrm{PO})_{l}$ the set of poles of $k(s)$, the set of poles of $k(s)$ that are to the right of $\Omega_{c}$, and the set of poles of $k(s)$ that are to the left of $\Omega_{c}$, respectively.

THEOREM 2.2. Let $\int_{\Omega_{c}} k(s) z^{-s} d s$ converge absolutely, that is, the conditions of Theorem 1.2 are satisfied.

(a) If $T \leq 0$, then

$$
\int_{\Omega_{c}} k(s) z^{-s} d s=-2 \pi i \sum_{s_{j} \in(\mathrm{PO})_{r}} z^{-s_{j}} \operatorname{Res}\left(k(s), s_{j}\right)
$$

and in case $T=0, z$ must also satisfy $|z|>\exp \left(-L+2 \pi \sum_{\operatorname{Re} \alpha_{j}<0}\left|\operatorname{Im} \alpha_{j}\right|\right)$.

(b) If $T \geq 0$, then

$$
\int_{\Omega_{c}} k(s) z^{-s} d s=2 \pi i \sum_{s_{j} \in(\mathrm{PO})_{l}} z^{-s_{j}} \operatorname{Res}\left(k(s), s_{j}\right) ;
$$

and in case $T=0, z$ must also satisfy $|z|<\exp \left(-L+\pi\left(\sum_{j=1}^{P}\left|\operatorname{Im} \alpha_{j}\right|-\sum_{j=1}^{Q}\left|\operatorname{Im} \beta_{j}\right|\right)-\right.$ $\left.2 \pi \sum_{\operatorname{Re} \alpha_{j}>0}\left|\operatorname{Im} \alpha_{j}\right|\right)$.

Proof. Making the change of variable $s \rightarrow s-c$, one sees that

$$
\int_{\Omega_{c}} k(s) z^{-s} d s=z^{-c} \int_{\Omega_{0}} k_{c}(s) z^{-s} d s, \quad k_{c}(s)=\frac{\prod_{j=1}^{P} \Gamma\left(p_{j}+c \alpha_{j}+\alpha_{j} s\right)}{\prod_{j=1}^{Q} \Gamma\left(q_{j}+c \beta_{j}+\beta_{j} s\right)},
$$

where the contour of integration $\Omega_{0}$ is the horizontal shifting of the contour $\Omega_{c}$ by $c$ units. The poles of $k_{c}(s)$ are precisely the horizontal shifting of the poles of $k(s)$ by $c$ units, and have the same position with respect to $\Omega_{0}$ as the corresponding poles of $k(s)$ with respect to $\Omega_{c}$; and moreover, if $s_{0}$ is a pole of $k(s)$, then $\operatorname{Res}_{s=s_{0}} k(s)=$ $\operatorname{Res}_{s=s_{0}-c} k_{c}(s)$. Thus, without a loss of generality we may assume that $c=0$.

We construct a family of circles with centers at the origin and whose radii $R_{r}$ approaching infinity. Moreover, we insure that the family stays a positive distance away from the set PO. The poles of $k(s)$ are lying on finitely many half-lines, and equidistant on each of these half-lines. Let $l_{1}, l_{2}, \ldots, l_{J}$ be those half-lines. Note that $J \leq P$. Let $z_{i}$ and $y_{i}$ be the initial point and the distance between consecutive poles on $l_{i}$, respectively, $i=1,2, \ldots, J$. For each positive integer $r$, the annulus $r-1 \leq|s| \leq r$, contains finitely many poles of $k(s)$ that are on $l_{i}$, say $M_{i}(r), i=1,2, \ldots, J$. If $Z_{1}^{i}(r)$ and $Z_{2}^{i}(r)$ are the points of intersections of $l_{i}$ with the circles $|s|=r-1$ and $|s|=r$, respectively, then

$$
\left(M_{i}(r)-1\right) y_{i} \leq\left|Z_{1}^{i}(r)-Z_{2}^{i}(r)\right|
$$

Now

$$
Z_{1}^{i}(r)=z_{i}+r_{1} e^{i \theta_{i}}, \quad Z_{2}^{i}(r)=z_{i}+r_{2} e^{i \theta_{i}},
$$

for some $r_{1}$ and $r_{2}$, and since

$$
r-1-\left|z_{i}\right|=\left|Z_{1}^{i}(r)\right|-\left|z_{i}\right| \leq r_{1}, \quad r_{2} \leq\left|Z_{2}^{i}(r)\right|+\left|z_{i}\right|=r+\left|z_{i}\right|,
$$


it follows that

$$
\left|Z_{1}^{i}(r)-Z_{2}^{i}(r)\right|=r_{2}-r_{1} \leq r+\left|z_{i}\right|-\left(r-1-\left|z_{i}\right|\right)=1+2\left|z_{i}\right| .
$$

Therefore,

$$
\sum_{i=1}^{J} M_{i}(r) \leq \sum_{i=1}^{J}\left(1+\frac{1}{y_{i}}\left|Z_{1}^{i}(r)-Z_{2}^{i}(r)\right|\right) \leq J+\sum_{i=1}^{J} \frac{1}{y_{i}}\left(1+2\left|z_{i}\right|\right)=v,
$$

which means that the total number of poles in the annulus $r-1 \leq|s| \leq r$ is bounded by the constant $v$ which is independent of $r$.

Let $s_{1}, s_{2}, \ldots, s_{J_{r}}$ be the poles of $k(s)$ on or within the annulus $r-1 \leq|s| \leq r$. Then $J_{r} \leq v$. Assume further that $r-1=s_{0} \leq\left|s_{1}\right| \leq\left|s_{2}\right| \leq \cdots \leq\left|s_{J_{r}}\right| \leq s_{J_{r}+1}=r$, and $s_{i_{0}} \in\left\{s_{0}, s_{1}, \ldots, s_{J_{r}}\right\}$ such that

$$
\left|s_{i_{0}+1}\right|-\left|s_{i_{0}}\right|=\max _{0 \leq i \leq J_{r}}\left\{\left|s_{i+1}\right|-\left|s_{i}\right|\right\} .
$$

Clearly,

$$
\left|s_{i_{0}+1}\right|-\left|s_{i_{0}}\right| \geq \frac{1}{v+1} .
$$

Let $R_{r}=(1 / 2)\left(\left|s_{i_{0}+1}\right|+\left|s_{i_{0}}\right|\right)$. If $s$ is such that $|s|=R_{r}$, then

$$
|w-s| \geq\left|R_{r}-\right| w|| \geq \frac{1}{2}\left(\left|s_{i_{0}+1}\right|-\left|s_{i_{0}}\right|\right) \geq \frac{1}{2(v+1)} \quad \forall w \in \mathrm{PO},
$$

and hence, the promised family of circles may be taken as all circles centered at the origin and whose radii are $R_{r}, r \geq 1$.

Let

$$
I_{C_{r}}=\frac{1}{2 \pi i} \int_{C_{r}} k(s) z^{-s} d s .
$$

The integral is taken in a clockwise direction around the contour $C_{r}$, consisting of the contours $C_{r}^{1}$ and $C_{r}^{2}$, where $C_{r}^{1}$ is a large circular arc, with center at the origin and radius $R_{r}$, lying to the right of $\Omega_{0}$ and originating from and ending on $\Omega_{0}$; and $C_{r}^{2}=\left\{s \in \Omega_{0}:|s| \leq R_{r}\right\}$. It is clear from the definition of $R_{r}$ that $C_{r}$ does not pass through any pole of $k(s)$. Now we split $I_{C_{r}}$ up into a sum of two integrals

$$
I_{C_{r}}=I_{C_{r}^{1}}+I_{C_{r}^{2}} .
$$

The integral $I_{C r}$ is equal to the negative of the sum of all the residues of $k(s) z^{-s}$ at its poles within the contour $C_{r}$ (due to the negative orientation of the contour $C_{r}$ ), that will cover $(\mathrm{PO})_{r}$ as $r \rightarrow \infty$, and since

$$
\frac{1}{2 \pi i} \int_{\Omega_{0}} k(s) z^{-s} d s=\lim _{r \rightarrow \infty} I_{C_{r}^{2}},
$$

Theorem 2.2(a) would be proved if we show that $\lim _{r \rightarrow \infty} I_{C_{r}^{1}}=0$. Since

$$
\left.\left|I_{C_{r}^{1}}\right| \leq \frac{1}{2} \max _{s \in C_{r}^{1}}\left|s k(s) z^{-s}\right| \quad \text { (for large } r\right), \quad d\left(\mathrm{PO}, C_{r}^{1}\right) \geq \frac{1}{2(1+v)},
$$


it follows that $\lim _{r \rightarrow \infty} I_{C_{r}^{1}}=0$, if we can show that $\lim _{|s| \rightarrow \infty}\left|s k(s) z^{-s}\right|=0$, for $\operatorname{Re}(s) \geq 0$, $d(s, \mathrm{PO}) \geq 1 /(2(1+v))$. Here $d(\mathbb{A}, \mathbb{B})$ denotes the distance between the two sets $\mathbb{A}$ and $\mathbb{B}$. For this purpose we use the asymptotic expansion of $|k(s)|$ given in Lemma 1.1.

We deduce that

$$
\begin{aligned}
f(s):= & \frac{\prod_{j=1}^{Q}\left|(1 / 2)\left(1+S\left(\beta_{j}, s\right)\right)+\left(1-S\left(\beta_{j}, s\right)\right) \sin \pi\left(q_{j}+\beta_{j} s\right)\right|}{\prod_{j=1}^{P}\left|(1 / 2)\left(1+S\left(\alpha_{j}, s\right)\right)+\left(1-S\left(\alpha_{j}, s\right)\right) \sin \pi\left(p_{j}+\alpha_{j} s\right)\right|} \\
= & \frac{\prod_{\operatorname{Re} \beta_{j}<0}\left|2 \sin \pi\left(q_{j}+\beta_{j} s\right)\right|}{\prod_{\operatorname{Re} \alpha_{j}<0}\left|2 \sin \pi\left(p_{j}+\alpha_{j} s\right)\right|} \\
& \times\left[\frac{1}{2}(1+\operatorname{signIm} s) \frac{\prod_{\operatorname{Re} \beta_{j}=0, \operatorname{Im} \beta_{j}>0}\left|2 \sin \pi\left(q_{j}+\beta_{j} s\right)\right|}{\prod_{\operatorname{Re} \alpha_{j}=0, \operatorname{Im} \alpha_{j}>0}\left|2 \sin \pi\left(p_{j}+\alpha_{j} s\right)\right|}\right. \\
& \left.+\frac{1}{2}(1-\operatorname{signIm} s) \frac{\prod_{\operatorname{Re} \beta_{j}=0, \operatorname{Im} \beta_{j}<0}\left|2 \sin \pi\left(q_{j}+\beta_{j} s\right)\right|}{\prod_{\operatorname{Re} \alpha_{j}=0, \operatorname{Im} \alpha_{j}<0}\left|2 \sin \pi\left(p_{j}+\alpha_{j} s\right)\right|}\right] .
\end{aligned}
$$

Our first objective is to obtain an upper estimate of $f(s)$ for all $s$ such that $\operatorname{Re} s \geq 0$, and $d(s, \mathrm{PO}) \geq 1 /(2(1+v))$. Then $\sin \pi\left(p_{j}+\alpha_{j} s\right) \neq 0$, and according to (2.1)

$$
\begin{aligned}
e^{-\pi\left|\operatorname{Im}\left(p_{j}+\alpha_{j} s\right)\right|}\left|\sin \pi\left(p_{j}+\alpha_{j} s\right)\right| & \geq \min \left\{\left|\alpha_{j}\right| d(s, \mathrm{PO}), \frac{1}{4}\right\} \\
& \geq \min \left\{\frac{\min _{j=1,2, \ldots, P}\left\{\left|\alpha_{j}\right|\right\}}{2(1+v)}, \frac{1}{4}\right\} .
\end{aligned}
$$

Since

$$
\begin{aligned}
& \left|\operatorname{Im}\left(\beta_{j} s\right)\right|-\left|\operatorname{Im}\left(\alpha_{j} s\right)\right| \\
& \quad \leq(\operatorname{Re} s)\left|\operatorname{Im} \beta_{j}\right|+|\operatorname{Im} s|\left|\operatorname{Re} \beta_{j}\right|-\left(|\operatorname{Im} s|\left|\operatorname{Re} \alpha_{j}\right|-(\operatorname{Re} s)\left|\operatorname{Im} \alpha_{j}\right|\right) \\
& \quad=\left(\left|\operatorname{Im} \beta_{j}\right|+\left|\operatorname{Im} \alpha_{j}\right|\right) \operatorname{Re} s+\left(\left|\operatorname{Re} \beta_{j}\right|-\left|\operatorname{Re} \alpha_{j}\right|\right)|\operatorname{Im} s|,
\end{aligned}
$$

we see that, through the use of (2.21) and the fact that $|\sin z| \leq e^{|\operatorname{Im} z|}$, for all $\operatorname{complex} z$,

$$
\begin{array}{r}
\frac{\prod_{\operatorname{Re} \beta_{j}<0}\left|2 \sin \pi\left(q_{j}+\beta_{j} s\right)\right|}{\prod_{\operatorname{Re} \alpha_{j}<0}\left|2 \sin \pi\left(p_{j}+\alpha_{j} s\right)\right|} \leq C \exp \left(\pi(\operatorname{Re} s)\left[\sum_{\operatorname{Re} \beta_{j}<0}\left|\operatorname{Im} \beta_{j}\right|+\sum_{\operatorname{Re} \alpha_{j}<0}\left|\operatorname{Im} \alpha_{j}\right|\right]\right. \\
\left.+\pi|\operatorname{Im} s|\left[\sum_{\operatorname{Re} \beta_{j}<0}\left|\operatorname{Re} \beta_{j}\right|-\sum_{\operatorname{Re} \alpha_{j}<0}\left|\operatorname{Re} \alpha_{j}\right|\right]\right) .
\end{array}
$$

Here and throughout the proof, $C$ denotes a universal positive constant, that may be distinct in different instances. Since $\operatorname{Im}\left(q_{j}+\beta_{j} s\right)=\operatorname{Im} q_{j}+\left(\operatorname{Im} \beta_{j}\right) \operatorname{Re} s$, if $\operatorname{Re} \beta_{j}=0$, we see that

$$
\begin{aligned}
& \frac{\prod_{\operatorname{Re} \beta_{j}=0, \operatorname{Im} \beta_{j}>0}\left|2 \sin \pi\left(q_{j}+\beta_{j} s\right)\right|}{\prod_{\operatorname{Re} \alpha_{j}=0, \operatorname{Im} \alpha_{j}>0}\left|2 \sin \pi\left(p_{j}+\alpha_{j} s\right)\right|} \\
& \quad \leq C\left(\exp \pi(\operatorname{Re} s)\left[\sum_{\operatorname{Re} \beta_{j}=0, \operatorname{Im} \beta_{j}>0}\left|\operatorname{Im} \beta_{j}\right|-\sum_{\operatorname{Re} \alpha_{j}=0, \operatorname{Im} \alpha_{j}>0}\left|\operatorname{Im} \alpha_{j}\right|\right]\right),
\end{aligned}
$$




$$
\begin{aligned}
& \frac{\prod_{\operatorname{Re} \beta_{j}=0, \operatorname{Im} \beta_{j}<0}\left|2 \sin \pi\left(q_{j}+\beta_{j} s\right)\right|}{\prod_{\operatorname{Re} \alpha_{j}=0, \operatorname{Im} \alpha_{j}<0}\left|2 \sin \pi\left(p_{j}+\alpha_{j} s\right)\right|} \\
& \quad \leq C \exp \left(\pi(\operatorname{Re} s)\left[\sum_{\operatorname{Re} \beta_{j}=0, \operatorname{Im} \beta_{j}<0}\left|\operatorname{Im} \beta_{j}\right|-\sum_{\operatorname{Re} \alpha_{j}=0, \operatorname{Im} \alpha_{j}<0}\left|\operatorname{Im} \alpha_{j}\right|\right]\right) .
\end{aligned}
$$

Combining (2.24) and (2.25) gives

$$
\begin{aligned}
\frac{1}{2}(1+\operatorname{signIm} s) \frac{\prod_{\operatorname{Re} \beta_{j}=0, \operatorname{Im} \beta_{j}>0}\left|2 \sin \pi\left(q_{j}+\beta_{j} s\right)\right|}{\prod_{\operatorname{Re} \alpha_{j}=0, \operatorname{Im} \alpha_{j}>0}\left|2 \sin \pi\left(p_{j}+\alpha_{j} s\right)\right|} \\
+\frac{1}{2}(1-\operatorname{signIm} s) \frac{\prod_{\operatorname{Re} \beta_{j}=0, \operatorname{Im} \beta_{j}<0}\left|2 \sin \pi\left(q_{j}+\beta_{j} s\right)\right|}{\prod_{\operatorname{Re} \alpha_{j}=0, \operatorname{Im} \alpha_{j}<0}\left|2 \sin \pi\left(p_{j}+\alpha_{j} s\right)\right|} \\
\leq C \exp \left(( \operatorname { R e } s ) \left[-\frac{\pi}{2}\left(\sum_{\operatorname{Re} \alpha_{j}=0}\left|\operatorname{Im} \alpha_{j}\right|-\sum_{\operatorname{Re} \beta_{j}=0}\left|\operatorname{Im} \beta_{j}\right|\right)\right.\right. \\
\left.\left.\quad-\frac{\pi}{2}(\operatorname{sign} \operatorname{Im} s)\left(\sum_{\operatorname{Re} \alpha_{j}=0} \operatorname{Im} \alpha_{j}-\sum_{\operatorname{Re} \beta_{j}=0} \operatorname{Im} \beta_{j}\right)\right]\right) .
\end{aligned}
$$

Using this estimate in the definition of $f(s)$, and observing that

$$
\begin{aligned}
-X & +2 \pi \sum_{\operatorname{Re} \alpha_{j}<0}\left|\operatorname{Im} \alpha_{j}\right| \\
& =\pi\left(\sum_{\operatorname{Re} \alpha_{j}<0}\left|\operatorname{Im} \alpha_{j}\right|+\sum_{\operatorname{Re} \beta_{j}<0}\left|\operatorname{Im} \beta_{j}\right|\right)-\frac{\pi}{2}\left(\sum_{\operatorname{Re} \alpha_{j}=0}\left|\operatorname{Im} \alpha_{j}\right|-\sum_{\operatorname{Re} \beta_{j}=0}\left|\operatorname{Im} \beta_{j}\right|\right), \\
N & =T-2 \sum_{\operatorname{Re} \beta_{j}<0}\left|\operatorname{Re} \beta_{j}\right|-\sum_{\operatorname{Re} \alpha_{j}<0}\left|\operatorname{Re} \alpha_{j}\right|,
\end{aligned}
$$

we obtain

$$
\begin{aligned}
f(s) \leq C \exp \left(\frac{\pi}{2}(T-N)|\operatorname{Im} s|+(\operatorname{Re} s)[\right. & -\frac{\pi}{2}(\operatorname{signIm} s)\left(\sum_{\operatorname{Re} \alpha_{j}=0} \operatorname{Im} \alpha_{j}-\sum_{\operatorname{Re} \beta_{j}=0} \operatorname{Im} \beta_{j}\right) \\
& \left.\left.-X+2 \pi \sum_{\operatorname{Re} \alpha_{j}<0}\left|\operatorname{Im} \alpha_{j}\right|\right]\right) .
\end{aligned}
$$

Since if $z \neq 0,\left|z^{-s}\right|=e^{-(\operatorname{Re} s) \ln |z|+(\operatorname{Im} s) \arg z}$, and $|Y(s)| \leq C$, then (2.28) and Lemma 1.1 yield

$$
\begin{aligned}
\left|s k(s) z^{-s}\right| \leq & C|s|^{\Delta_{0}+1} \times \exp \left((\operatorname{Im} s)(\arg z-M-T \operatorname{Arg} s)+\frac{\pi}{2}(T-N)|\operatorname{Im} s|\right) \\
& \times \exp \left((\operatorname{Re} s)\left[-L-T+T \ln |s|-\ln |z|+2 \pi \sum_{\operatorname{Re} \alpha_{j}<0}\left|\operatorname{Im} \alpha_{j}\right|\right]\right),
\end{aligned}
$$

for $\operatorname{Re} s \geq 0$, and $d(s, \mathrm{PO}) \geq 1 /(2(1+v))$. 
Let

$$
\begin{gathered}
w(s)=-T\left(\frac{\pi}{2}-|\operatorname{Arg} s|\right), \quad \Theta(s)=\frac{\pi}{2} N-(\operatorname{sign} \operatorname{Im} s)(\arg z-M), \\
V=-L-T-\ln |z|+2 \pi \sum_{\operatorname{Re} \alpha_{j}<0}\left|\operatorname{Im} \alpha_{j}\right|,
\end{gathered}
$$

and note that

$$
\Theta(s) \geq b=\min \left\{\frac{\pi}{2} N \pm(\arg z-M)\right\} \geq 0,
$$

since by assumption, the conditions of Theorem 1.2 are satisfied. Moreover,

$$
(\operatorname{Im} s)(\arg z-M-T \operatorname{Arg} s)+\frac{\pi}{2}(T-N)|\operatorname{Im} s|=-|\operatorname{Im} s|[w(s)+\Theta(s)],
$$

and (2.29) can be rewritten in the form

$$
\left|s k(s) z^{-s}\right| \leq C|s|^{\Delta_{0}+1} e^{-|\operatorname{Im} s|[w(s)+\Theta(s)]} e^{(\operatorname{Re} s)[V+T \ln |s|]} .
$$

Armed with (2.33), we now proceed to establish part (a) of the theorem. Let

$$
\begin{aligned}
& R_{1}=\left\{s: d(s, \mathrm{PO}) \geq \frac{1}{2(1+v)}, \frac{\pi}{4} \leq|\operatorname{Arg} s| \leq \frac{\pi}{2}\right\}, \\
& R_{2}=\left\{s: d(s, \mathrm{PO}) \geq \frac{1}{2(1+v)},|\operatorname{Arg} s|<\frac{\pi}{4}\right\},
\end{aligned}
$$

and notice that,

$$
\begin{gathered}
|\operatorname{Im} s| \geq \frac{|s|}{\sqrt{2}}, \quad s \in R_{1}, \\
\operatorname{Re} s>\frac{|s|}{\sqrt{2}}, \quad s \in R_{2} .
\end{gathered}
$$

(a1) Assume that $T<0$.

We consider two subcases:

(i) $|\arg z-M|<(\pi / 2) N$.

In this case $b>0$. Since $T<0,(V+T \ln |s|) \rightarrow-\infty$, as $|s| \rightarrow \infty$, and hence, the function $e^{(\operatorname{Re} s)[V+T \ln |s|]}$ is bounded for $\operatorname{Re} s \geq 0$. On the other hand, $w(s)=|T|(\pi / 2-$ $|\operatorname{Arg} s|) \geq 0, \Theta(s) \geq b>0$, and for all $s$ in $R_{1},|\operatorname{Im} s| \geq(1 / \sqrt{2})|s|$. Thus,

$$
\left|s k(s) z^{-s}\right| \leq C|s|^{\Delta_{0}+1} e^{-(b / \sqrt{2})|s|}, \quad s \in R_{1} .
$$

The assumption $T<0$ implies $\lim _{|s| \rightarrow \infty}[V+T \ln |s|]=-\infty$, and consequently, $V+$ $T \ln |s|<-1$ as $|s| \rightarrow \infty$. Since $\operatorname{Re} s>(|s| / \sqrt{2}), s \in R_{2}$, it follows that

$$
\left|s k(s) z^{-s}\right| \leq C|s|^{\Delta_{0}+1} e^{(\operatorname{Re} s)[V+T \ln |s|]} \leq C|s|^{\Delta_{0}+1} e^{-(1 / \sqrt{2})|s|}, \quad s \in R_{2} .
$$

From (2.37) and (2.38) it immediately follows that $\lim _{|s| \rightarrow \infty}\left|s k(s) z^{-s}\right|=0$, for $\operatorname{Re} s \geq 0$ and $d(s, \mathrm{PO}) \geq 1 /(2(1+v))$.

(ii) $|\arg z-M|=(\pi / 2) N$.

In this case, necessarily $\Delta_{0}<-1$ because the convergence conditions of Theorem 1.2 are satisfied. Thus, $|s|^{\Delta_{0}+1} \rightarrow 0$, as $|s| \rightarrow \infty$. Since the functions $e^{-|\operatorname{Im} s|[w(s)+\Theta(s)]}$, 
and $e^{(\operatorname{Re} s)[V+T \ln |s|]}$ are bounded for all $\operatorname{Re} s \geq 0$, it follows from (2.33) that $\lim _{|s| \rightarrow \infty}\left|s k(s) z^{-s}\right|=0$, for Re $s \geq 0$ and $d(s, \mathrm{PO}) \geq 1 /(2(1+v))$.

(a2) Assume $T=0$, and $z$ satisfies $|z|>\exp \left(-L+2 \pi \sum_{\operatorname{Re} \alpha_{j}<0}\left|\operatorname{Im} \alpha_{j}\right|\right)$, in addition to the conditions of Theorem 1.2.

In this case $w(s)=0$ for all $s$, and we again consider two subcases:

(i) $|\arg z-M|<(\pi / 2) N$.

As before, $\Theta(s) \geq b>0$. The condition on $z$ implies that $V<0$, and thus

$$
[V+T \ln |s|] \operatorname{Re} s=V \operatorname{Re} s \leq 0, \quad \operatorname{Re} s \geq 0 .
$$

It follows from this, (2.33), (2.35), and (2.36) that

$$
\begin{aligned}
& \left|s k(s) z^{-s}\right| \leq C|s|^{\Delta_{0}+1} e^{-(b / \sqrt{2})|s|}, \quad s \in R_{1}, \\
& \left|s k(s) z^{-s}\right| \leq C|s|^{\Delta_{0}+1} e^{-(|V| / \sqrt{2})|s|}, \quad s \in R_{2} .
\end{aligned}
$$

It immediately follows from (2.40) and (2.41) that $\lim _{|s| \rightarrow \infty}\left|s k(s) z^{-s}\right|=0$, for $\operatorname{Re} s \geq 0$ and $d(s, \mathrm{PO}) \geq 1 /(2(1+v))$.

(ii) $|\arg z-M|=(\pi / 2) N$.

In this case, necessarily $\Delta_{0}<-1$ because the convergence conditions of Theorem 1.2 are satisfied. Thus, $|s|^{\Delta_{0}+1} \rightarrow 0$, as $|s| \rightarrow \infty$, and since the functions $e^{-\Theta(s)|\operatorname{Im} s|}$, and $e^{V \operatorname{Re} s}$ are bounded for all Re $s \geq 0$, it follows from (2.33) that $\left|s k(s) z^{-s}\right| \rightarrow 0$, as $|s| \rightarrow \infty$, for $\operatorname{Re} s \geq 0$ and $d(s, \mathrm{PO}) \geq 1 /(2(1+v))$. This completes the proof of Theorem 2.2(a).

Now we proceed to prove Theorem 2.2(b).

Let $\alpha_{j}^{\prime}=-\alpha_{j}, j=1,2, \ldots, P ; \beta_{j}^{\prime}=-\beta_{j}, j=1,2, \ldots, Q$, and define

$$
k_{1}(s)=\frac{\prod_{j=1}^{P} \Gamma\left(p_{j}+\alpha_{j}^{\prime} s\right)}{\prod_{j=1}^{Q} \Gamma\left(q_{j}+\beta_{j}^{\prime} s\right)} .
$$

Then under the change of variables $s \rightarrow-s$,

$$
\int_{\Omega_{c}} k(s) z^{-s} d s=-\int_{\Omega_{-c}^{\prime}} k(-s)\left(\frac{1}{z}\right)^{-s} d s=\int_{-\Omega_{-c}^{\prime}} k_{1}(s)\left(\frac{1}{z}\right)^{-s} d s,
$$

where $\Omega_{-c}^{\prime}$ is the reflection of $\Omega_{c}$ about the origin. Observe that $-\Omega_{-c}^{\prime}$ is directed from $-c-i \infty$ to $-c+i \infty$. We want to apply part (a) to $k_{1}(s)$ and the contour $-\Omega_{-c}^{\prime}$. For this purpose, let $T^{\prime}, M^{\prime}, L^{\prime}$, and $N^{\prime}$ denote the constants associated with $k_{1}(s)$ that are computed as in Lemma 1.1 and Theorem 1.2, respectively. It is easy to see that

$$
T^{\prime}=-T, \quad N^{\prime}=N, \quad M^{\prime}=-M .
$$

However,

$$
\begin{aligned}
L^{\prime} & =\sum_{j=1}^{P}\left[\left(\operatorname{Arg} \alpha_{j}^{\prime}\right) \operatorname{Im} \alpha_{j}^{\prime}-\left(\ln \left|\alpha_{j}^{\prime}\right|\right) \operatorname{Re} \alpha_{j}^{\prime}\right]-\sum_{j=1}^{Q}\left[\left(\operatorname{Arg} \beta_{j}^{\prime}\right) \operatorname{Im} \beta_{j}^{\prime}-\left(\ln \left|\beta_{j}^{\prime}\right|\right) \operatorname{Re} \beta_{j}^{\prime}\right] \\
& =-L+\pi\left[\sum_{j=1}^{P}\left|\operatorname{Im} \alpha_{j}\right|-\sum_{j=1}^{Q}\left|\operatorname{Im} \beta_{j}\right|\right] .
\end{aligned}
$$


Hence,

$$
\left|M^{\prime}-\arg \left(\frac{1}{z}\right)\right|=|M-\arg (z)|, \quad \text { for any } z,
$$

and $T \geq 0$ if and only if $T^{\prime} \leq 0$. It is clear that $s \in(\mathrm{PO})_{l}$ if and only if $-s$ is a pole of $k_{1}(s)$, that is, to the right of $-\Omega_{-c}^{\prime}$. Furthermore, if $s_{0}$ is a pole of $k_{1}(s)$ of order $m$, then there is a $\rho>0$ such that

$$
k_{1}(s)=f(s)+\sum_{j=1}^{m} \frac{a_{-j}}{\left(s-s_{0}\right)^{j}}, \text { for }\left|s-s_{0}\right|<\rho,
$$

where $a_{-m} \neq 0$ and $f$ is analytic at $s_{0}$. Hence

$$
k(s)=f(-s)+\sum_{j=1}^{m}(-1)^{j} \frac{a_{-j}}{\left(s+s_{0}\right)^{j}}, \text { for }\left|s+s_{0}\right|<\rho,
$$

and therefore

$$
\operatorname{Res}\left(k(s),-s_{0}\right)=-\operatorname{Res}\left(k_{1}(s), s_{0}\right) \text {. }
$$

Thus, if $T>0$, then

$$
\begin{aligned}
\int_{\Omega_{c}} k(s) z^{-s} d s & =\int_{-\Omega_{-c}^{\prime}} k_{1}(s)\left(\frac{1}{z}\right)^{-s} d s=-2 \pi i \sum_{s_{j} \in(\mathrm{PO})_{l}}\left(\frac{1}{z}\right)^{-s_{j}} \operatorname{Res}\left(k_{1}(s),-s_{j}\right) \\
& =2 \pi i \sum_{s_{j} \in(\mathrm{PO})_{l}} z^{-s_{j}} \operatorname{Res}\left(k(s), s_{j}\right) .
\end{aligned}
$$

In case $T=0$, then $T^{\prime}=0$, and $z$ must also satisfy

$$
\frac{1}{|z|}>\exp \left(-L^{\prime}+2 \pi \sum_{\operatorname{Re} \alpha_{j}^{\prime}<0}\left|\operatorname{Im} \alpha_{j}^{\prime}\right|\right)
$$

or, equivalently,

$$
|z|<\exp \left(-L+\pi\left(\sum_{j=1}^{P}\left|\operatorname{Im} \alpha_{j}\right|-\sum_{j=1}^{Q}\left|\operatorname{Im} \beta_{j}\right|\right)-2 \pi \sum_{\operatorname{Re} \alpha_{j}>0}\left|\operatorname{Im} \alpha_{j}\right|\right) .
$$

This completes the proof of Theorem 2.2.

REMARK 2.3. If $\mu=0, N>0$, and $T \neq 0$, then the $H$-function with complex parameters is analytic in the sector $|\arg z-M|<(\pi / 2) N$. This is the case since the series of the residues at the left poles, if $T>0$, and the series of the residues at the right poles, if $T<0$, represent analytic functions.

REMARK 2.4. If $\sum_{\operatorname{Re} \alpha_{j}=0}\left|\operatorname{Im} \alpha_{j}\right|>\sum_{j=1}^{Q}\left|\operatorname{Im} \beta_{j}\right|+\sum_{\operatorname{Re} \alpha_{j} \neq 0}\left|\operatorname{Im} \alpha_{j}\right|$, then

$$
\begin{aligned}
r_{1} & =\exp \left(-L+2 \pi \sum_{\operatorname{Re} \alpha_{j}<0}\left|\operatorname{Im} \alpha_{j}\right|\right) \\
& <\exp \left(-L+\pi\left(\sum_{j=1}^{P}\left|\operatorname{Im} \alpha_{j}\right|-\sum_{j=1}^{Q}\left|\operatorname{Im} \beta_{j}\right|\right)-2 \pi \sum_{\operatorname{Re} \alpha_{j}>0}\left|\operatorname{Im} \alpha_{j}\right|\right)=r_{2} .
\end{aligned}
$$


Thus, if $T=0$, then the $H$-function with complex parameters can be computed for any $z$ in the annulus $r_{1}<|z|<r_{2}$ by the residues at the right poles as well as the residues at the left poles. This situation has no analogy in the real case, that is, when $(\alpha)_{P}$ and $(\beta)_{Q}$ are real vectors.

3. Some special cases of the $H$-function. In general, if $w$ and $\alpha \neq 0$ are complex numbers, then

$$
\operatorname{Res}_{s=-(m+w) / \alpha} \Gamma(w+\alpha s)=\frac{(-1)^{m}}{\alpha(m !)}, \quad m=1,2, \ldots
$$

EXAMPLE 3.1. Let $a_{1}, a_{2}, \ldots, a_{P+1} ; b_{1}, b_{2}, \ldots, b_{P}$ be complex numbers such that $\sum_{j=1}^{P+1} \operatorname{Re} a_{j}<\sum_{j=1}^{P} \operatorname{Re} b_{j}$. Then

$$
\begin{aligned}
{ }_{P+1} F_{P}\left((a)_{P+1} ;(b)_{P} ;(-1)^{P} z\right)= & i \operatorname{signIm}(z) \prod_{j=1}^{P+1} \Gamma\left(1-a_{j}\right) \prod_{j=1}^{P} \Gamma\left(b_{j}\right) \\
& \times H_{2 P+1}^{1}\left(z^{i}, c \mid \begin{array}{l}
(0, i) \\
\left(1-a_{j}, i\right)_{P+1},\left(b_{j},-i\right)_{P}
\end{array}\right),
\end{aligned}
$$

$|z|=1$, where $c>0$ if $\operatorname{Im} z \geq 0, c<0$ if $\operatorname{Im} z<0$, and ${ }_{p} F_{q}(z)$ is the generalized hypergeometric function (see [2, 4, 18]).

In fact for the function

$$
\begin{aligned}
& H_{2 P+1}^{1}\left(x, c \mid \begin{array}{l}
(0, i) \\
\left(1-a_{j}, i\right)_{P+1},\left(b_{j},-i\right)_{P}
\end{array}\right) \\
&=\frac{1}{2 \pi i} \int_{c-i \infty}^{c+i \infty} \frac{\Gamma(i s)}{\prod_{j=1}^{P+1} \Gamma\left(1-a_{j}+i s\right) \prod_{j=1}^{P} \Gamma\left(b_{j}-i s\right)} x^{-s} d s .
\end{aligned}
$$

it is easy to see that $N=0, M=0$, and

$$
\Delta_{c}=P-\sum_{j=1}^{P+1} \operatorname{Re}\left(1-a_{j}\right)-\sum_{j=1}^{P} \operatorname{Re} b_{j}=-1+\sum_{j=1}^{P+1} \operatorname{Re} a_{j}-\sum_{j=1}^{P} \operatorname{Re} b_{j}<-1,
$$

so the conditions of Theorem 1.2 are satisfied.

Since $L=0$ and $T=0$, we can compute the value of the function using the residues at the poles $s=i m, m=0,1,2, \ldots$.

If $c>0$, then the poles are left poles. Hence, computing with left poles, for $0<x \leq 1$,

$$
\begin{aligned}
H_{2 P+1}^{1} & \left(x, c \mid \begin{array}{l}
(0, i) \\
\left(1-a_{j}, i\right)_{P+1},\left(b_{j},-i\right)_{P}
\end{array}\right) \\
= & -i \sum_{m=0}^{\infty} \frac{(-1)^{m}}{m !} \frac{x^{-i m}}{\prod_{j=1}^{P+1} \Gamma\left(1-a_{j}-m\right) \prod_{j=1}^{P} \Gamma\left(b_{j}+m\right)} \\
= & -i \sum_{m=0}^{\infty} \frac{(-1)^{m P}}{m !} \frac{\prod_{j=1}^{P+1}\left(a_{j}\right)_{m}}{\prod_{j=1}^{P+1} \Gamma\left(1-a_{j}\right)} \frac{x^{-i m}}{\prod_{j=1}^{P} \Gamma\left(b_{j}\right) \prod_{j=1}^{P}\left(b_{j}\right)_{m}} \\
= & -\frac{i_{P+1} F_{P}\left((a)_{P+1} ;(b)_{P} ;(-1)^{P} x^{-i}\right)}{\prod_{j=1}^{P+1} \Gamma\left(1-a_{j}\right) \prod_{j=1}^{P} \Gamma\left(b_{j}\right)} .
\end{aligned}
$$


Let $z=x^{-i}$, then $|z|=1, x=z^{i}$, and we obtain (3.2) for $\operatorname{Im}(z) \geq 0$.

If $c<0$, then the poles are right poles. Hence, computing with right poles, for $x>1$,

$$
H_{2 P+1}^{1}\left(x, c \mid \begin{array}{l}
(0, i) \\
\left(1-a_{j}, i\right)_{P+1},\left(b_{j},-i\right)_{P}
\end{array}\right)=\frac{i_{P+1} F_{P}\left((a)_{P+1} ;(b)_{P} ;(-1)^{P} x^{-i}\right)}{\prod_{j=1}^{P+1} \Gamma\left(1-a_{j}\right) \prod_{j=1}^{P} \Gamma\left(b_{j}\right)} .
$$

Let $z=x^{-i}$, then $|z|=1, x=z^{i}$, and we obtain (3.2) for $\operatorname{Im}(z)<0$.

REMARK 3.2. It seems to the authors that (3.2) provides the first integral representation for the generalized hypergeometric geometric function ${ }_{P+1} F_{P}(z)$ on the unit circle.

EXAMPLE 3.3. If $\operatorname{Re} p \neq 0,1+\operatorname{Re} p<\operatorname{Re} q$, and $\operatorname{Im} p>0$, then

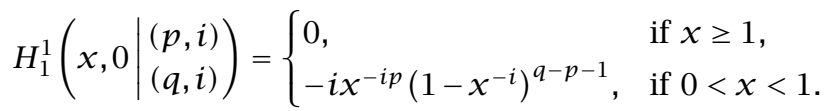

Since $N=0=M$ and $\Delta_{0}=\operatorname{Re} p-\operatorname{Re} q<-1$, the $H$-function exists for $x>0$. Since $T=0=L$, the function can be computed by the residues at the right poles for $x>1$, and by the residues at the left poles for $0<x<1$. The poles are at $s=i m+i p$, $m=0,1,2, \ldots$. Since $\operatorname{Im} p>0$, all the poles are on the left, and hence

$$
H_{1}^{1}\left(\begin{array}{l|l}
x, 0 & \left.\begin{array}{c}
(p, i) \\
(q, i)
\end{array}\right)=0, \quad \text { if } x>1
\end{array}\right.
$$

and for $0<x<1$,

$$
\begin{aligned}
H_{1}^{1}\left(x, 0 \mid \begin{array}{c}
(p, i) \\
(q, i)
\end{array}\right) & =\sum_{m=0}^{\infty}\left[\operatorname{Res}_{s=i(m+p)} \Gamma(p+i s)\right] \frac{1}{\Gamma(q-p-m)} x^{-i m-i p} \\
& =-i \sum_{m=0}^{\infty} \frac{(-1)^{m}}{(m !) \Gamma(q-p-m)} x^{-i m-i p}=-i x^{-i p}\left(1-x^{-i}\right)^{q-p-1} .
\end{aligned}
$$

EXAMPLE 3.4. If $p$ and $q$ are complex numbers such that $\operatorname{Re} p, \operatorname{Re} q \neq 0$, and $\operatorname{Re} p+$ $\operatorname{Re} q<0$, then

$$
H_{0}^{2}(x, 0 \mid(p, i),(q,-i))= \begin{cases}0, & \text { if } \operatorname{Im}(p) \operatorname{Im}(q)<0, \\ \frac{-i(\operatorname{sgn} \operatorname{Im} p) \Gamma(p+q)}{x^{-i q}\left(1+x^{i}\right)^{p+q}}, & \text { if } \operatorname{Im}(p) \operatorname{Im}(q)>0,\end{cases}
$$

for all $x>0$.

It is easy to check that $M=N=0$, and $\Delta_{0}=-1+\operatorname{Re} p+\operatorname{Re} q<-1$. Hence,

$$
H_{0}^{2}(x, 0 \mid(p, i),(q,-i))=\frac{1}{2 \pi i} \int_{-i \infty}^{i \infty} \Gamma(p+i s) \Gamma(q-i s) x^{-s} d s
$$

exists for all $x>0$.

Since $T=0$ and $L=\pi$, the function can be computed using the residues at the right poles of $\Gamma(p+i s) \Gamma(q-i s) x^{-s}$ for $x>e^{-\pi}$ and the residues at the left poles for 
$0<x<e^{\pi}$. Thus in the interval $\left(e^{-\pi}, e^{\pi}\right)$, the function can be computed using the residues at the right as well as at the left poles.

The poles of $\Gamma(p+i s)$ are at $s=i m+i p$, while those of $\Gamma(q-i s)$ are at $s=-i m-i q$, and

$$
\operatorname{Res}_{s=i(m+p)} \Gamma(p+i s)=-i \frac{(-1)^{m}}{m !}, \quad \operatorname{Res}_{s=-i(m+q)} \Gamma(q-i s)=i \frac{(-1)^{m}}{m !} .
$$

(i) The case $\operatorname{Im} p>0, \operatorname{Im} q<0$.

In this case all the poles are left poles and hence

$$
H_{0}^{2}(x, 0 \mid(p, i),(q,-i))=0, \text { for } x>e^{-\pi},
$$

and for $0<x<e^{\pi}$,

$$
\begin{aligned}
& H_{0}^{2}(x, 0 \mid(p, i),(q,-i) \\
&= \sum_{m=0}^{\infty}\left[\operatorname{Res}_{s=i(m+p)} \Gamma(p+i s)\right] \Gamma(p+q+m) x^{-i m-i p} \\
&+\sum_{m=0}^{\infty}\left[\operatorname{Res}_{s=-i(m+q)} \Gamma(q-i s)\right] \Gamma(p+q+m) x^{i m+i q} \\
&=-i \Gamma(p+q) \sum_{m=0}^{\infty} \frac{(-1)^{m}}{m !}(p+q)_{m} x^{-i m-i p}+i \Gamma(p+q) \sum_{m=0}^{\infty} \frac{(-1)^{m}}{m !}(p+q)_{m} x^{i m+i q} \\
&=-i \Gamma(p+q) x^{-i p}\left(1+x^{-i}\right)^{-p-q}+i \Gamma(p+q) x^{i q}\left(1+x^{i}\right)^{-p-q}=0 .
\end{aligned}
$$

(ii) The case $\operatorname{Im} p<0, \operatorname{Im} q>0$.

In this case all the poles are right poles and hence,

$$
H_{0}^{2}(x, 0 \mid(p, i),(q,-i))=0, \text { for } 0<x<e^{\pi},
$$

and for $x>e^{-\pi}$, the exact computation as above shows that,

$$
H_{0}^{2}\left(x,\left.0\right|^{(p, i),(q,-i)}\right)=0, \text { for } x>e^{-\pi} .
$$

(iii) The case $\operatorname{Im} p<0, \operatorname{Im} q<0$.

In this case, the poles at $s=i m+i p$ are right poles, and the poles at $s=-i m-i q$ are left poles. Thus, for $x>e^{-\pi}$,

$$
\begin{aligned}
& H_{0}^{2}(x, 0 \mid(p, i),(q,-i)) \\
& \quad=-\sum_{m=0}^{\infty}\left[\operatorname{Res}_{s=i(m+p)} \Gamma(p+i s)\right] \Gamma(p+q+m) x^{-i m-i p} \\
& \quad=i \Gamma(p+q) \sum_{m=0}^{\infty} \frac{(-1)^{m}}{m !}(p+q)_{m} x^{-i m-i p} \\
& \quad=i \Gamma(p+q) x^{-i p}\left(1+x^{-i}\right)^{-p-q}=\frac{i \Gamma(p+q)}{x^{-i q}\left(1+x^{i}\right)^{p+q}},
\end{aligned}
$$


and for $0<x<e^{\pi}$,

$$
\begin{aligned}
& H_{0}^{2}(x, 0 \mid(p, i),(q,-i)) \\
& \quad=\sum_{m=0}^{\infty}\left[\operatorname{Res}_{s=-i(m+q)} \Gamma(q-i s)\right] \Gamma(p+q+m) x^{i m+i q} \\
& \quad=i \Gamma(p+q) \sum_{m=0}^{\infty} \frac{(-1)^{m}}{m !}(p+q)_{m} x^{i m+i q}=i \Gamma(p+q) x^{i q}\left(1+x^{i}\right)^{-p-q} .
\end{aligned}
$$

Therefore,

$$
H_{0}^{2}\left(x,\left.0\right|^{(p, i),(q,-i)}\right)=i \Gamma(p+q) x^{i q}\left(1+x^{i}\right)^{-p-q}, \quad \forall x>0 .
$$

(iv) The case $\operatorname{Im} p>0, \operatorname{Im} q>0$.

In this case, the poles at $s=i m+i p$ are left poles, and the poles at $s=-i m-i q$ are right poles. Thus, for $0<x<e^{\pi}$,

$$
\begin{aligned}
& H_{0}^{2}(x, 0 \mid(p, i),(q,-i)) \\
& \quad=\sum_{m=0}^{\infty}\left[\operatorname{Res}_{s=i(m+p)} \Gamma(p+i s)\right] \Gamma(p+q+m) x^{-i m-i p} \\
& \quad=-i \Gamma(p+q) \sum_{m=0}^{\infty} \frac{(-1)^{m}}{m !}(p+q)_{m} x^{-i m-i p} \\
& \quad=-i \Gamma(p+q) x^{-i p}\left(1+x^{-i}\right)^{-p-q}=-i \Gamma(p+q) x^{i q}\left(1+x^{i}\right)^{-p-q},
\end{aligned}
$$

and for $x>e^{-\pi}$,

$$
\begin{aligned}
& H_{0}^{2}(x, 0 \mid(p, i),(q,-i)) \\
& \quad=-\sum_{m=0}^{\infty}\left[\operatorname{Res}_{s=-i(m+q)} \Gamma(q-i s)\right] \Gamma(p+q+m) x^{i m+i q} \\
& \quad=-i \Gamma(p+q) \sum_{m=0}^{\infty} \frac{(-1)^{m}}{m !}(p+q)_{m} x^{i m+i q}=-i \Gamma(p+q) x^{i q}\left(1+x^{i}\right)^{-p-q} .
\end{aligned}
$$

Therefore,

$$
H_{0}^{2}(x, 0 \mid(p, i),(q,-i))=-i \Gamma(p+q) x^{i q}\left(1+x^{i}\right)^{-p-q}, \quad \forall x>0 .
$$

REMARK 3.5. The special case $x=1$ of the previous example is evaluated in [6, formula 6.411].

EXAMPLE 3.6. If $a$ is a complex number, then

$$
H_{2}^{1}\left(z, c \mid \begin{array}{l}
(0,1) \\
(i a,-i),(1-i a, i)
\end{array}\right)=\frac{1}{2 \pi}\left(e^{\pi a-e^{\pi} z}-e^{-\pi a-e^{-\pi} z}\right), \quad c>0,|\arg z|<\frac{\pi}{2} .
$$


It is easy to check that $N=1, M=0$, and the conditions for existence are satisfied for $|\arg z|<\pi / 2$. Since $T=1>0$, we can compute the function using the left poles at $s=-m, m=0,1,2, \ldots$. Thus,

$$
\begin{aligned}
H_{2}^{1}\left(z, c \mid \begin{array}{l}
(0,1) \\
(i a,-i),(1-i a, i)
\end{array}\right) & =\frac{1}{2 \pi i} \int_{c-i \infty}^{c+i \infty} \frac{\Gamma(s)}{\Gamma(i a-i s) \Gamma(1-i a+i s)} z^{-s} d s \\
& =\sum_{m=0}^{\infty} \frac{(-1)^{m}}{m !} \cdot \frac{1}{\Gamma(i a+i m) \Gamma(1-i a-i m)} z^{m} \\
& =\frac{1}{\pi} \sum_{m=0}^{\infty} \frac{(-1)^{m} \sin \pi i(a+m)}{m !} z^{m} \\
& =\frac{1}{\pi} \sum_{m=0}^{\infty} \frac{(-1)^{m} \sinh \pi(a+m)}{m !} z^{m} \\
& =\frac{e^{a \pi}}{2 \pi} \sum_{m=0}^{\infty} \frac{\left(-e^{\pi} z\right)^{m}}{m !}-\frac{e^{-a \pi}}{2 \pi} \sum_{m=0}^{\infty} \frac{\left(-e^{-\pi} z\right)^{m}}{m !} \\
& =\frac{1}{2 \pi}\left(e^{\pi a-e^{\pi} z}-e^{-\pi a-e^{-\pi} z}\right) .
\end{aligned}
$$

EXAMPLE 3.7. If $c>0$ and $a$ is a complex number, $\operatorname{Re} a>c$, then

$$
H_{0}^{3}(z, c \mid(0,1),(i a,-i),(1-i a, i))=\pi \sum_{m=0}^{\infty} \frac{(-z)^{m}}{m ! \sinh \pi(a+m)}, \quad|\arg z|<\frac{\pi}{2} .
$$

Now, $N=1, M=0$, and the conditions of convergence are satisfied for $|\arg z|<\pi / 2$. Since $T=1>0$, we can compute the value of the function using the residues at the left poles of the function $\Gamma(i a-i s) \Gamma(1-i a+i s) \Gamma(s) z^{-s}$. Because $\operatorname{Re} a>c>0$, the only left poles we have are the poles $s=-m$ of $\Gamma(s)$. Hence,

$$
\begin{aligned}
H_{0}^{3}(z, c \mid(0,1),(i a,-i),(1-i a, i)) & =\frac{1}{2 \pi i} \int_{c-i \infty}^{c+i \infty} \Gamma(i a-i s) \Gamma(1-i a+i s) \Gamma(s) z^{-s} d s \\
& =\sum_{m=0}^{\infty} \frac{(-1)^{m}}{m !} \Gamma(i a+i m) \Gamma(1-i a-i m) z^{m} \\
& =\sum_{m=0}^{\infty} \frac{(-1)^{m}}{m !} \cdot \frac{\pi}{\sin \pi i(a+m)} z^{m} \\
& =\pi \sum_{m=0}^{\infty} \frac{(-z)^{m}}{m ! \sinh \pi(a+m)} .
\end{aligned}
$$

EXAMPLE 3.8. Let $a$ and $b$ be complex numbers such that $1+\operatorname{Re} a<\operatorname{Re} b$, and $\operatorname{Im} a>-1 / 2$, then

$$
H_{1}^{1}\left(x, \frac{1}{2} \mid \begin{array}{ll}
(a, i) \\
(b, i)
\end{array}\right)= \begin{cases}-\frac{i}{\Gamma(b-a)} x^{-i a}\left(1-x^{-i}\right)^{b-a-1}, & \text { if } 0<x<1, \\
0, & \text { if } x>1 .\end{cases}
$$

It is easy to see that $N=0=M, \Delta_{1 / 2}=\operatorname{Re} a-\operatorname{Re} b<-1$, and the conditions of Theorem 1.2 are fulfilled. Moreover, $T=0, L=0$, and the poles are at $s=i a+i m$, 
$m=0,1,2, \ldots$. Since $\operatorname{Im} a>-1 / 2$, there are no right poles, and therefore

$$
H_{1}^{1}\left(x, \frac{1}{2} \mid \begin{array}{l}
(a, i) \\
(b, i)
\end{array}\right)=0, \text { for } x>1 .
$$

For $0<x<1$,

$$
H_{1}^{1}\left(x, \frac{1}{2} \mid \begin{array}{c}
(a, i) \\
(b, i)
\end{array}\right)=-i \sum_{m=0}^{\infty} \frac{(-1)^{m}}{m ! \Gamma(b-a-m)} x^{-i a-i m}=-\frac{i}{\Gamma(b-a)} x^{-i a}\left(1-x^{-i}\right)^{b-a-1} .
$$

ACKNOWLEDGement. The authors wish to thank Professor H. M. Srivastava for the helpful comments. Fadhel A. Al-Musallam was partially supported by Kuwait University Research Administration under grant SM 167.

\section{REFERENCES}

[1] F. A. Al-Musallam and V. K. Tuan, $H$-function with complex parameters I: existence, Int. J. Math. Math. Sci. 25 (2001), no. 9, 571-586.

[2] W. N. Bailey, Generalized Hypergeometric Series, Cambridge Tracts in Mathematics and Mathematical Physics, no. 32, Stechert-Hafner, New York, 1964. MR 32\#2625.

[3] B. L. J. Braaksma, Asymptotic expansions and analytic continuations for a class of Barnesintegrals, Compositio Math. 15 (1963), 239-341. MR 29\#4923. Zbl 129.28604.

[4] A. Erdélyi, W. Magnus, F. Oberhettinger, and F. G. Tricomi, Higher Transcendental Functions, vol. I, McGraw-Hill, New York, 1953. MR 15,419i. Zbl 051.30303.

[5] C. Fox, The $G$ and $H$ functions as symmetrical Fourier kernels, Trans. Amer. Math. Soc. 98 (1961), 395-429. MR 24\#A1427. Zbl 096.30804.

[6] I. S. Gradshteyn and I. M. Ryzhik, Table of Integrals, Series, and Products, Academic Press [Harcourt Brace Jovanovich Publishers], New York, 1980. MR 81g:33001. Zbl 521.33001.

[7] A. M. Mathai, Distributions of Test Statistics: Exact and Asymptotic, Null and Non-null, Methods/Comparisons/Research Frontiers, The American Sciences Press Series in Mathematical and Management Sciences, vol. 18, American Sciences Press, Inc., Columbus, 1989, reprinted from American Journal of Mathematical and Management Sciences 9 (1989), no. 1-2. Zbl 743.62017.

[8] A. M. Mathai and H. J. Haubold, Modern Problems in Nuclear and Neutrion Astrophysics, Akademie-Verlag, Berlin, 1988.

[9] A. M. Mathai and R. K. Saxena, Generalized Hypergeometric Functions with Applications in Statistics and Physical Sciences, Lecture Notes in Mathematics, vol. 348, SpringerVerlag, Berlin, 1973. MR 57\#3471. Zbl 272.33001.

[10] _ The H-function with Applications in Statistics and Other Disciplines, Halsted Press [John Wiley \& Sons], New York, 1978. MR 80a:33007.

[11] C. S. Meijer, On the G-function. I, Nederl. Akad. Wetensch. Proc. 49 (1946), 227-237 Indag. Math. 8 (1946), 124-134. MR 8,156a. Zbl 060.19901.

[12] _ On the G-function. II, Nederl. Akad. Wetensch. Proc. 49 (1946), 344-356 Indag. Math. 8 (1946), 213-225. MR 8,156b. Zbl 060.19901.

[13] _ On the G-function. III, Nederl. Akad. Wetensch. Proc. 49 (1946), 457-469 Indag. Math. 8 (1946), 312-324. MR 8,156c. Zbl 060.19901.

[14] _ On the G-function. IV, Nederl. Akad. Wetensch. Proc. 49 (1946), 632-641 Indag. Math. 8 (1946), 391-400. MR 8,156d. Zbl 060.19901.

[15] - On the G-function. V, VI, Nederl. Akad. Wetensch. Proc. 49 (1946), 765-772, 936943 Indag. Math. 8 (1946), 468-475, 595-602. MR 8,379c. Zbl 060.19901.

[16] _ On the G-function. VII, VIII, Nederl. Akad. Wetensch. Proc. 49 (1946), 1063-1072, 1165-1175 Indag. Math. 8 (1946), 661-670, 713-723. MR 8,379d. Zbl 060.19901. 
[17] A. P. Prudnikov, Y. A. Brychkov, and O. I. Marichev, Integrals and Series, Nauka, Moscow, 1986 (Russian), Supplementary Chapters. MR 88f:00012. Zbl 606.33001.

[18] L. J. Slater, Generalized Hypergeometric Functions, Cambridge University Press, Cambridge, 1966. MR 34\#1570. Zbl 135.28101.

[19] H. M. Srivastava, K. C. Gupta, and S. P. Goyal, The H-functions of One and Two Variables, South Asian Publishers Pvt. Ltd., New Delhi, 1982, with applications.

FAdHel A. Al-Musallam: Department of MATHEMATics AND Computer SCIENCE, KuWAit UNIVERSITY, P.O. BOX 5969 SAFAT, 13060, KUWAIT

E-mail address: musa17am@math-1.sci . kuniv. edu. kw

Vu Kim TuAN: DEPARTMENT OF MATHEMATICS AND COMPUTER SCIENCE, KuWAIT UNIVERSITY, P.O. BOX 5969 SAFAT, 13060, KUWAIT

E-mail address: vu@math-1. sci . kuniv. edu. kw 


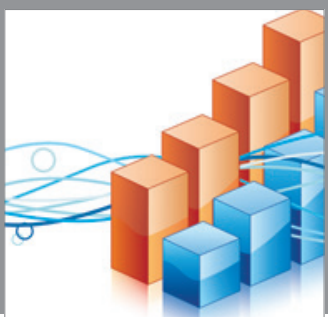

Advances in

Operations Research

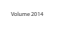

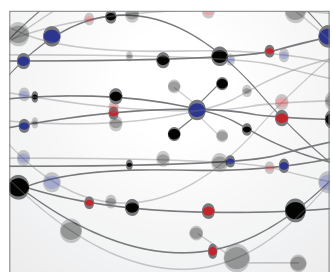

\section{The Scientific} World Journal
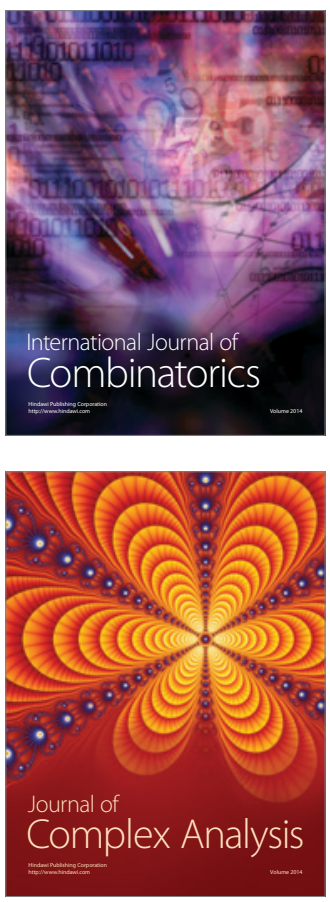

International Journal of

Mathematics and

Mathematical

Sciences
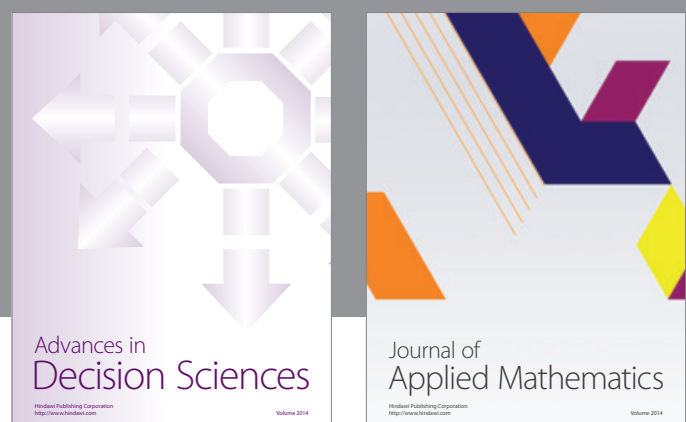

Journal of

Applied Mathematics
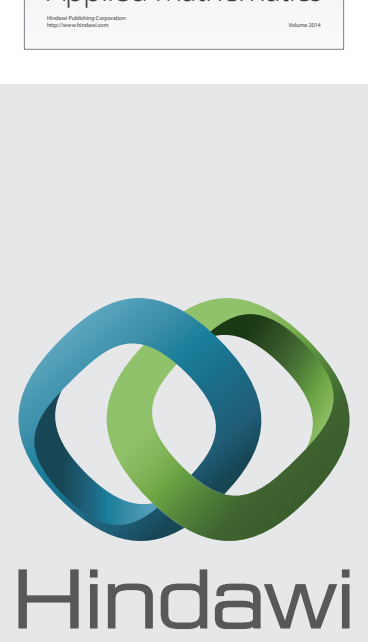

Submit your manuscripts at http://www.hindawi.com
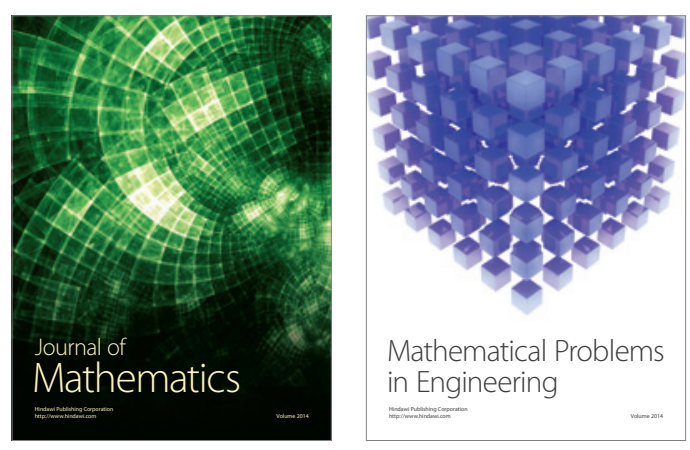

Mathematical Problems in Engineering
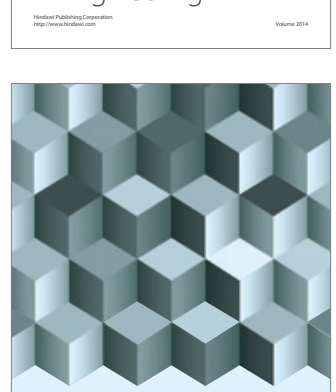

Journal of

Function Spaces
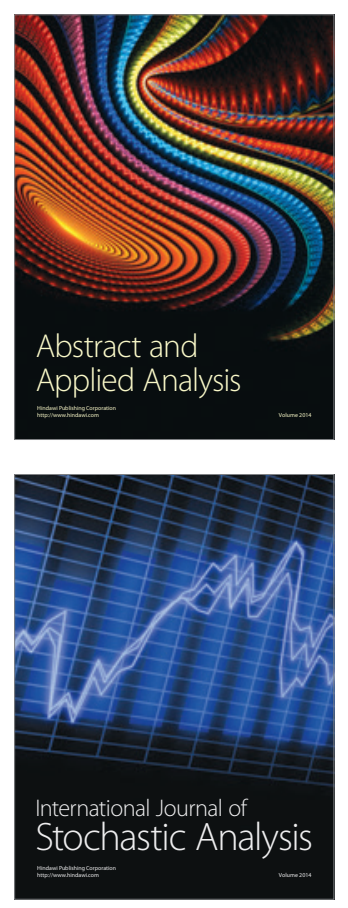

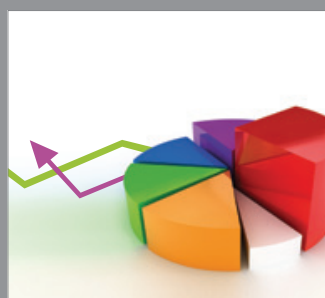

ournal of

Probability and Statistics

Promensencen
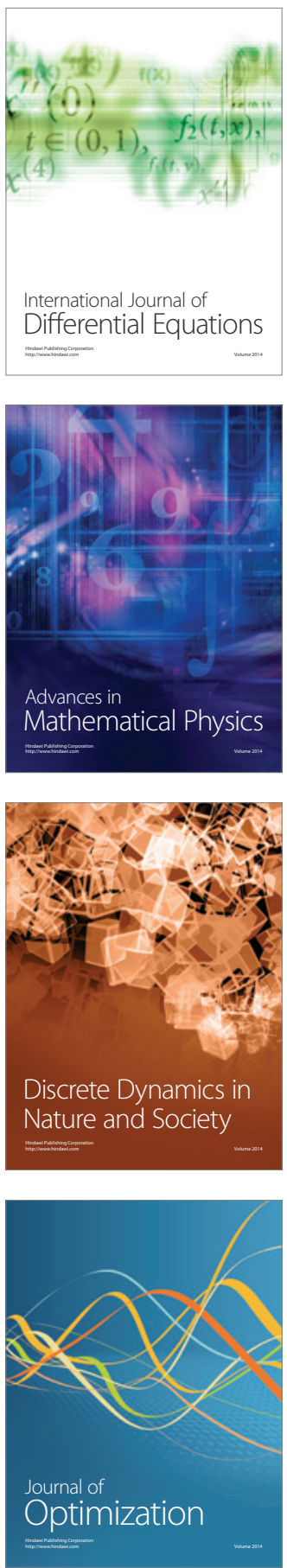